\title{
POSITRON EMISSION TOMOGRAPHY WITH 2-[18F]- FLUORO-2-DEOXY-D-GLUCOSE FOR INITIAL STAGING OF HODGKIN LYMPHOMA: A SINGLE CENTER EXPERIENCE IN BRAZIL
}

\author{
Juliano Julio Cerci,' Luís Fernando Pracchia, ${ }^{I I}$ José Soares Junior,' Camila da \\ Cruz Gouveia Linardi, II José Claudio Meneghetti, I Valeria Buccheri II
}

doi: 10.1590/S1807-59322009000600002

Cerci JJ, Pracchia LF, Soares-Junior J, Linardi CCG, Meneghetti JC, Buccheri V. Positron emission tomography with 2-[18f]-Fluoro-2-Deoxy-d-Glucose for initial staging of hodgkin lymphoma: a single center experience in brazil. Clinics. 2009;64(6):491-8.

BACKGROUND: 2-[18F]-Fluoro-2-Deoxy-D-Glucose (FDG-PET) is a well established functional imaging modality for the initial staging of Hodgkin lymphoma (HL) in patients from Western Europe and North America. The reliability of FDG-PET in populations of different ethnic groups is unclear, as all investigations published to date have come from developed countries.

PURPOSE: The aim of the present study was to investigate the effectiveness of FDG-PET in the initial staging of HL patients in a Brazilian population.

METHODS: Eighty-two patients with newly diagnosed HL were prospectively included in the study. All patients were staged with both conventional clinical staging (CCS) methods, including computed tomography (CT) and whole-body FDG-PET methods. A standard of reference for the nodal regions and the extranodal organs was determined using all available information, including the CCS methods, FDG-PET, the diagnostic histology and the follow-up examinations. The results of the CCS were then compared to the FDG-PET results.

RESULTS: The sensitivity of FDG-PET was higher for nodal staging than that of CT ( $87.8 \%$ vs. $61.6 \%$, respectively). FDG-PET was also more sensitive than CT in regard to evaluating the extranodal organs for lymphomatous involvement (96.2\% vs. $40.0 \%$, respectively). FDG-PET detected all 16 patients who were characterized by a positive bone marrow biopsy and identified an additional 4 patients with bone marrow disease. The incorporation of FDG-PET coupled with CCS in the staging procedure upstaged $20 \%(17 / 82)$ of the patients and downstaged $11 \%(9 / 82)$ of the patients. As a result of these changes in staging, 15\% (13/82) of the patients would have received a different therapeutic regimen.

CONCLUSIONS: The FDG-PET method is superior to CT for the detection of nodal and extra-nodal HL. The observation that the FDG-PET method upstaged the disease was the most common result (20\% of patients) brought about by the addition of PET to the staging algorithm, even in a population of patients with a high incidence of advanced disease. However, changes in stages based on FDG-PET results should be confirmed by biopsy.

KEYWORDS: FDG-PET; computed tomography; Hodgkin; lymphoma; initial staging.

\section{INTRODUCTION}

Over the last several decades, the Hodgkin lymphoma
(HL) cure rates have remained among the highest in oncologic patients, mainly due to the improvement of treatment strategies, which include chemotherapy and radiotherapy. A precise staging of HL patients is essential for selecting an appropriate treatment, as well as for determining the prognosis. The Ann-Arbor system modified in Cotswolds ${ }^{1}$ is widely used to define the conventional clinical stage (CCS) of HL patients.

Conventional staging procedures include a physical examination, computed tomography (CT), and a bone 
marrow (BM) biopsy. Although CT provides important anatomic details, it cannot reliably distinguish between malignant and benign lesions because the definition of nodal or organ involvement is based only on morphological criteria. The correlation of CT findings with pathological staging demonstrated that the size of the lymph nodes was not always associated with the presence of $\mathrm{HL} .{ }^{2}$ In addition, CT has a limited sensitivity for the detection of splenic, hepatic and bone marrow involvement. Therefore, the clinical staging of patients may be underestimated and thus lead to inappropriate therapy, or clinical staging may overestimate the severity and lead to overtreatment.

In recent years, positron emission tomography (PET) with 2-[18F]-fluoro-2-Deoxy-D-glucose (FDG) has become an established modality for staging HL in the United States and Europe. ${ }^{3-6}$ Besides HL staging, FDG-PET is also useful for evaluating the treatment response and the posttreatment remission status of HL patients, both of which are matters beyond the scope of this paper. Guidelines for its use in lymphomas were recently proposed by an international group. ${ }^{7}$ However, the spectrum of HL varies across different ethnic populations and environments, with different frequencies of Epstein Barr Virus detectable in the tumor, different grades of disease aggressiveness and later diagnoses. In developing countries, such as Brazil, the incidence of the advanced stages is considerably higher than in the United States and Europe. ${ }^{8}$ Although upstaging of the disease is the most common result when PET is integrated into the staging procedure, the performance of FDG-PET staging in populations with a high frequency of advanced HL stages is still unknown.

\section{MATERIAL AND METHODS}

\section{Patients}

Eighty-two consecutive patients presenting newly diagnosed, biopsy proven, classical HL from the Hematology Division of the São Paulo University Clinics Hospital were eligible for this prospective study performed between August 2005 and January 2008. The only exclusion criterion was pregnancy. Notably, none of the patients had diabetes mellitus. The study was approved by the Ethical Board of the São Paulo University Clinics Hospital, and written informed consent was obtained from all participating patients.

\section{Conventional staging}

All patients were subjected to CCS procedures that included a physical examination, complete blood cell counts, blood chemistry analysis, CT scans (cervical, thoracic, abdomen and pelvic), and a bilateral BM biopsy. CT scans were sectioned at a thickness of $5 \mathrm{~mm}$, and oral and intravenous contrast agents were administered to all patients. The nodal or organ involvement, as determined by CT, was defined by the modified Ann Arbor criteria. ${ }^{1}$ All patients had undergone a BM biopsy performed at the posterior iliac crest. Bone marrow involvement was defined as positive only for cases in which the presence of Reed-Sternberg cells or its variants were unequivocally observed. The CCS of each patient was assigned according to the Ann Arbor staging system. ${ }^{1}$

\section{FDG-PET Imaging}

Whole-body PET images were acquired following a 60-minute uptake period after the intravenous administration of 296 to $444 \mathrm{MBq}$ ( 8 to $12 \mathrm{mCi}$ ) of FDG. Imaging was then performed via 2-D acquisition with a GE Advance PET scanner (General Electric Medical Systems Advance; Milwaukee, Wisconsin, USA). Attenuation correction was performed using ${ }^{68} \mathrm{Ge}$ sources. Prior to FDG administration, the fasting glucose level was measured in order to verify a blood glucose level $<160 \mathrm{mg} / \mathrm{dL}$. Diazepam was given orally to the majority of patients in order to avoid muscular and brown fat uptake.

Visual interpretation of the images was performed independently by two experienced observers who were not aware of the CCS results. Areas of nonphysiological abnormalities increased the FDG uptake over the background and were classified as positive for the disease. Nodal and extranodal lymphoma involvement was defined according to the criteria established by the Consensus of the Imaging Subcommittee of International Harmonization Project in Lymphoma. $^{7}$

The time between the staging CT and PET scan was no longer than two weeks.

\section{Treatment}

All patients were treated based on the CCS, regardless of the FDG-PET results. Stage I and II patients were treated with four to six cycles of the ABVD regimen (doxorubicin, bleomycin, vinblastine and dacarbazine). Stage III patients were treated with six to eight cycles of ABVD. Stage IV patients were treated with eight cycles of ABVD. Radiotherapy was included in all stage I or II patients and patients with bulky disease, regardless of their stage.

\section{Data analysis}

In order to establish the diagnostic accuracy of the 
FDG-PET and the CT results obtained by both methods in all of the nodal regions and organs, these results had to be compared to a gold standard technique, such as histological examination. However, for obvious practical and ethical reasons, this was not possible. Instead of the histological gold standard, a reference standard was used for this analysis. The reference standard for all nodal regions and organs was obtained by combining the initial FDG-PET and CT results with the results of the FDG-PET performed after the second chemotherapy cycle and CT scans performed after the fourth cycle of chemotherapy. If the initial FDG-PET and CT scans were positive, the region was defined as a true positive for lymphoma involvement, while negative findings gleaned from both of these initial methods were regarded as a true negative. Discordant cases were retrospectively analyzed by an interdisciplinary team consisting of radiologists, nuclear physicians and hemato-oncologists. The status of a region or organ was determined using data from an interim FDGPET performed after the second chemotherapy cycle, CT scans performed after the fourth cycle, CT and/or FDG-PET analyses obtained at the end of treatment, and, if available, a biopsy of the suspected lesion. For example, if a small lesion $(<1 \mathrm{~cm})$ was radiologically normal and the PET positive lymph node changed to PET negative during follow-up scans, this lesion was labeled as "committed." Alternatively, a large lymph node was considered "not committed" if it was PET negative and did not shrink during treatment while other enlarged nodes regressed and were PET negative at the end of treatment. To study BM involvement, the FDG-PET results were compared with the results of the bilateral BM biopsy. Notably, all of these patients had at least one year of follow-up evaluation after the completion of chemotherapy.

In the analysis of clinical stage, the results of both methods were compared (CCS and FDG-PET alone). This analysis indicated that the PET would lead to a change in the clinical strategy if the patient stage moved from an early to advanced disease stage and vice-versa (either I or II up to III or IV or III or IV down to I or II).

The diagnostic accuracies are given in terms of sensitivity, specificity, positive predictive value (PPV), and negative predictive value (NPV). SPSS 10.0 for Windows (SPSS Inc, Chicago, IL) was used for the statistical analysis.

\section{RESULTS}

\section{Patients}

Of the 82 patients evaluated, 40 were male. The median age at diagnosis was 32 years (16 to 82 years), and the most frequent histological subtype was nodular sclerosis, which was observed in 45 (54.8\%) of the patients. The demographic and clinical characteristics of the patients as well as their corresponding CCS methods are listed in Table 1.

Table 1 - Demographic and clinical characteristics of the Hodgkin's lymphoma patients

\begin{tabular}{|c|c|}
\hline Characteristics & $\mathrm{N}=82(\%)$ \\
\hline \multicolumn{2}{|l|}{ Sex } \\
\hline Female & $42(51.2 \%)$ \\
\hline Male & $40(48.8 \%)$ \\
\hline Age - median (range) & 32 (16 to 82$)$ \\
\hline \multicolumn{2}{|l|}{ Race } \\
\hline White & $39(47.5 \%)$ \\
\hline Black & $2(0.2 \%)$ \\
\hline Asian & $1(0.1 \%)$ \\
\hline Mixed & $40(48.7 \%)$ \\
\hline \multicolumn{2}{|l|}{ Pathological subtype } \\
\hline Nodular sclerosis & $45(54.8 \%)$ \\
\hline Mixed cellularity & $22(26.8 \%)$ \\
\hline Lymphocyte rich & $3(3.7 \%)$ \\
\hline Lymphocyte-depleted & $3(3.7 \%)$ \\
\hline Not classified & $9(11.0 \%)$ \\
\hline \multicolumn{2}{|l|}{ Ann Arbor Clinical Stage } \\
\hline I & $8(9.7 \%)$ \\
\hline II & $23(28.0 \%)$ \\
\hline III & $22(26.9 \%)$ \\
\hline IV & $29(35.4 \%)$ \\
\hline B Symptoms & $\mathbf{5 0}(60.9 \%)$ \\
\hline Bulky Disease & $48(58.5 \%)$ \\
\hline
\end{tabular}

\section{Staging accuracy in nodal sites}

A total of 535 nodal regions had initial involvement according to the reference standard. CT scans could detect HL in 336 of the 535 regions analyzed, and FDGPET detected involvement in 477 of the 535 regions. The observed frequency of nodal disease as a function of the site is listed in Table 2. The overall sensitivity, specificity, PPV and NPV for nodal staging of CT and FDG- PET/CT are provided in Table 4.

\section{Staging accuracy in extranodal sites}

The spleen, liver, lungs, bone marrow/bone and soft tissue were evaluated. HL was observed in 43 of the 92 regions analyzed (46.7\%) using $\mathrm{CT}$ and 86 of the 92 regions (93.4\%) using FDG-PET. The frequency of involvement as a function of the site is listed in Table 3. Table 4 shows the sensitivity, specificity, PPV and NPV of the CT and the FDG-PET methods for detection of organ involvement.

\section{PET Comparison to BM Biopsy}

Of the 82 patients, bilateral BM biopsy indicated marrow infiltration in 16 (19.5\%) patients. Notably, all of these cases 
Table 2 - Region-based analysis of lymph nodes sites using $\mathrm{CT}$ and FDG-PET in 82 patients during the initial staging

\begin{tabular}{lccc}
\hline Sites & $\begin{array}{c}\text { Reference } \\
\text { Standard }\end{array}$ & FDG-PET & TC \\
\hline Cervical & 113 & 100 & 66 \\
Supraclavicular & 107 & 96 & 68 \\
Mediastinal & 69 & 68 & 65 \\
Axillarys & 55 & 49 & 42 \\
Pulmonar Hilar & 56 & 44 & 24 \\
Paraortic/Mesen- & 61 & 53 & 42 \\
teric & & 17 & 7 \\
Iliac & 17 & 31 & 12 \\
Inguinal & 32 & 19 & 10 \\
Others & 25 & $\mathbf{4 7 7}$ & $\mathbf{3 3 6}$ \\
\hline TOTAL & $\mathbf{5 3 5}$ & &
\end{tabular}

Table 3 - Region-based analysis of extranodal sites using CT and FDG-PET in 82 patients during initial staging

\begin{tabular}{lccc}
\hline Sites & $\begin{array}{c}\text { Reference Stan- } \\
\text { dard }\end{array}$ & FDG-PET & TC \\
\hline Lungs & 29 & 26 & 22 \\
Spleen & 31 & 30 & 14 \\
Liver & 8 & 6 & 5 \\
Bone/Bone Marrow* & 21 & 21 & 1 \\
Soft Tissue & 3 & 3 & 1 \\
\hline TOTAL & $\mathbf{9 2}$ & $\mathbf{8 6}$ & $\mathbf{4 3}$ \\
\hline
\end{tabular}

* Bones includes bone marrow involvement.

Table 4 - Assessment of the overall accuracy rates using CT and FDG-PET on nodal and extranodal sites

\begin{tabular}{lcccc}
\hline & \multicolumn{2}{c}{ Nodal sites } & \multicolumn{2}{c}{ Extranodal sites } \\
\hline & PET & TC & PET & TC \\
\hline Sensitivity & $87.8 \%$ & $61.6 \%$ & $96.2 \%$ & $40.0 \%$ \\
Specificity & $98.8 \%$ & $99.0 \%$ & $99.5 \%$ & $98.7 \%$ \\
PPV* & $98.5 \%$ & $98.2 \%$ & $97.4 \%$ & $86.4 \%$ \\
NPV* & $90.3 \%$ & $74.8 \%$ & $99.2 \%$ & $86.4 \%$ \\
\hline
\end{tabular}

* PPV: positive predictive value; NPV: negative predictive value.

had multiple focal bone uptake of FDG, including the iliac crest region. Five other patients had a positive scan in the bone/BM as observed using FDG-PET in conjunction with negative BM biopsy results. Three patients had positive results in multiple bone/bone marrow areas including the posterior iliac crest FDG uptake, while one patient had a positive FDG uptake in multiple bone/BM areas but only limited uptake in the posterior iliac crest. The other patient had only one focal area of uptake located on a costal arch.
Of the five patients with PET positive scans in the bone/ $\mathrm{BM}$, the latter case was considered as a false positive due to the fracture of a costal bone observed on the CT results. Regarding the former four patients, two had magnetic resonance imaging results indicating focal $\mathrm{BM}$ alterations compatible with lymphoma infiltration, while another patient had bone involvement detected on a bone scintigraphy. In all of these patients, the bone/BM FDG-avid lesions disappeared in the interim PET scan performed after the second cycle of chemotherapy.

\section{Change in clinical stage and therapy}

Upon comparison of both methods used in the staging of patients, the method that included the FDG-PET was concordant with CCS in $56(68.2 \%)$ of the patients. In these concordant cases, additional committed lymph nodes were identified by FDG-PET in $48(85.7 \%)$ of the patients. In 26 $(31.7 \%)$ patients, the results obtained using FDG-PET scans were discordant with CCS. Seventeen patients (20.7\%) were upstaged, while nine patients (10.9\%) were downstaged. Of the seventeen upstaged patients, two moved from clinical stage I to II, one patient moved from stage I to IV, three patients moved from stage II to III, three patients moved from stage II to IV, and eight patients moved from stage III to IV. Of the nine downstaged patients, one moved from clinical stage II to I, four patients moved from stage III to II, one patient moved from stage IV to I, one patient moved from stage IV to II, and two patients moved from stage IV to III (Table 5).

If the FDG-PET results were included in the initial staging procedure, $13(15.8 \%)$ of the 82 patients would have had a change in the therapy strategy. The status of seven patients would have been modified from early to advanced stages, and six patients would have been modified from advanced to early stages. Consequently these patients would have received a more or less aggressive treatment regimen, respectively (Table 5).

As an example, Figure 1 shows images of a patient upstaged after FDG-PET analysis from stage II to III. The PET images demonstrated that FDG-PET positive foci in the spleen were not observed in the CT. While the biopsies of these lesions were not performed, the FDG positivity in the spleen disappeared when an early interim FDG-PET was completed after two cycles of chemotherapy (ABVD). Therefore, these images were considered FDG-PET true positive and $\mathrm{CT}$ false negative.

\section{DISCUSSION}

The reliability of FDG-PET in populations of different ethnic groups has not been investigated, as all studies 
Table 5 - Initial staging distribution for Conventional Clinical Staging (CCS) and FDG-PET according to the Ann Arbor staging system and the corresponding up- and down-staging

\begin{tabular}{|c|c|c|c|c|c|}
\hline & PET Stage I & PET Stage II & PET Stage III & PET Stage IV & $\begin{array}{c}\text { Total CCS Stage } \\
\left(\mathrm{n}^{\circ} \text { of cases }\right)\end{array}$ \\
\hline CCS Stage I & 5 & 2 & 0 & 1 & I (8) \\
\hline CCS Stage II & 1 & 16 & 3 & 3 & II (23) \\
\hline CCS Stage III & 0 & 4 & 10 & 8 & III (22) \\
\hline CCS Stage IV & 1 & 1 & 2 & 25 & IV (29) \\
\hline $\begin{array}{l}\text { Total PET Stage } \\
\left(n^{\circ} \text { of cases }\right)\end{array}$ & I (7) & II (23) & III (15) & IV (37) & 82 \\
\hline
\end{tabular}

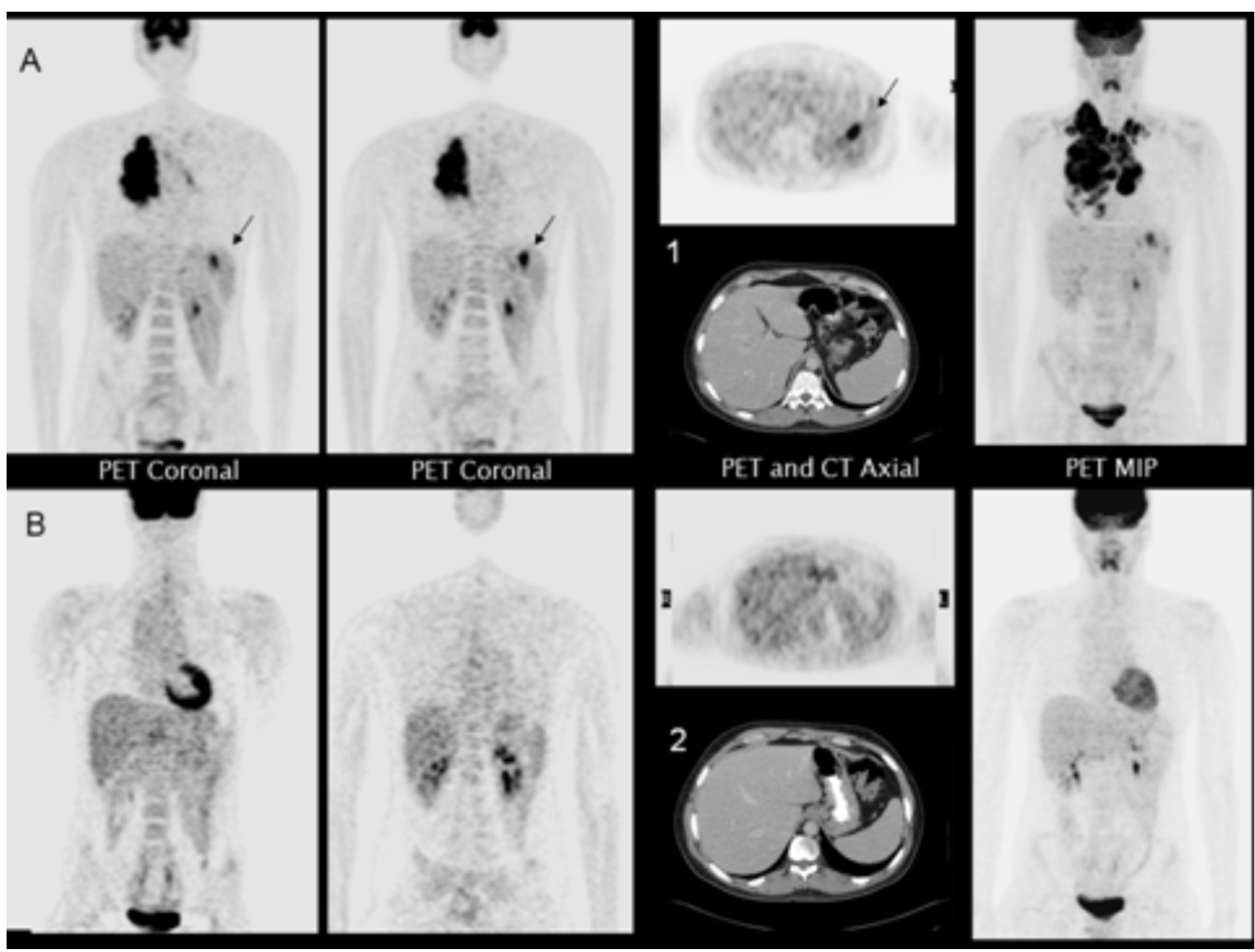

Figure 1 - A 22-year old male patient with conventional clinical stage II disease. A) FDG-PET coronal, axial and maximum intensity projection (MIP) images showed additional splenic lesions (arrows) not observed in the CT axial image (1). B) After two ABVD cycles, all lesions, including the splenic foci, disappeared; normal CT axial image (2).

published to date came from Western Europe and North America. In this unselected patient cohort, there was a high frequency of advanced stages of the disease, mainly stage IV ( $35.4 \%$ by conventional staging and $43.9 \%$ by FDG-PET). This particularly distinctive stage distribution provided an opportunity to compare the usefulness of FDG-PET with conventional methods not only for staging nodal regions but also for extranodal sites.

Although the most important indication of FDG-PET is in the evaluation of the treatment response and posttreatment evaluation of HL, all of which are matters beyond the scope of this paper, FDG-PET may be used in the initial 
staging and may help classify patients into the appropriate stages of the disease, which can thus change the type and duration of the administered therapy.

In this study, FDG-PET had a higher sensitivity than CT for the detection of nodal sites $(87.8 \%$ vs. $61.6 \%$, respectively), in agreement with other reports. ${ }^{3-6,10-13}$ Metabolic testing with fluorine-18-fluorodeoxyglucose has also been described for detecting residual tumor or recurrence. ${ }^{14}$ The sensitivity of PET for detecting organ involvement was also higher than that determined for CT $(96.2 \%$ vs. $40.0 \%$, respectively), particularly for the evaluation of splenic involvement. The modified AnnArbor system indicates that spleen infiltration is positive only when palpable splenomegaly is present or when there is radiological evidence gleaned from the CT results that are indicative of the presence of multiple focal defects. For detecting spleen lesions, the higher PET sensitivity compared to CT is probably related to the ability of FDGPET to detect metabolic changes in areas involved with HL before anatomical changes become visible. ${ }^{13}$

The diagnostic accuracy of FDG-PET for the detection of bone/BM involvement was also high when compared with results from the BM biopsy. FDG-PET identified all patients with a positive BM biopsy. In addition, another five patients were identified as positive using FDG-PET in bone/bone marrow sites but showed negative results in the BM biopsy. Only one of these discordant cases was considered as a false positive FDG-PET result due to the presence of a bone fracture. In all other discordant cases, the BM FDG-positive foci observed at staging disappeared during treatment. This discrepancy can be explained due to the existence of focal marrow infiltration by HL and biopsy sampling errors, even with bilateral sampling. As a consequence, the focal iliac $\mathrm{BM}$ involvement or infiltration at locations other than the posterior iliac crest could be missed by BM biopsy. Early studies have also demonstrated the usefulness of FDG-PET in the evaluation of BM involvement in lymphoma. ${ }^{15-18}$ In a recent meta-analysis, a good correlation was demonstrated between the results from FDG-PET and the BM biopsy in regard to the detection of $\mathrm{BM}$ involvement during the staging of patients with malignant lymphoma. However, the study involved a mixed population of different lymphoma types that have distinct patterns of BM infiltration and FDG avidity. ${ }^{19}$ Therefore, there is no consensus in regard to the idea that FDG-PET significantly reduces the need for BM biopsies as an initial staging procedure in HL stages II to IV. ${ }^{7}$

Computed tomography is the most commonly used imaging modality for staging HL due to its widespread availability and relatively low cost. ${ }^{12}$ Therefore, CT remains the standard imaging modality for initial staging of malignant lymphoma. However, an important limitation of $\mathrm{CT}$ is its failure to detect pathological changes in normalsized structures and lesions that have poor contrast with the surrounding tissue. An additional drawback of $\mathrm{CT}$ is that this method is not reliable for the detection of BM disease. ${ }^{12,20}$

Within recent years, FDG-PET has become the most important nuclear medicine and radiology imaging modality in the management of lymphoma. FDG-PET detects more disease sites and involved organs than conventional staging procedures, including $\mathrm{CT}$, and it has a large influence on staging. ${ }^{12,20,21}$

The major drawback of all studies that invoke FDGPET analyses for the staging of HL is the absence of a histological confirmation of all the FDG-avid lesions, which then requires the use of a reference standard instead of the gold standard. This limitation must also be considered in our study, due to the possibility of biasing the results in favor of PET. Consequently, caution must be invoked for cases that are upstaged by FDG-PET because it is well known that benign processes may cause FDG uptake, such as rebound thymic hyperplasia, infection, inflammation and muscular or brown fat uptake. ${ }^{7}$ It is also important to consider the normal bio-distribution of FDG and the lack of anatomical detail of PET scans alone. The FDG-PET/ CT fusion, using a combined PET/CT scanner, provides an accurate coregistration between the two modalities, allows for optimal interpretation of both modalities, and produces more accurate localization of the foci with increased FDG uptake than the stand-alone PET; this fusion may reduce the problems of physiological FDG uptake being misinterpreted as pathological and false localization of the disease. ${ }^{12}$ However, the results of this study are similar to the studies reported using PET/CT equipment. ${ }^{9}$

Another limitation of the current study is the analysis of a patient population from a single health center. Several authors have extensively studied the performance of PET and PET/CT compared with CT over the past five years; as such, the compilation of multiple studies with larger series is required to substantiate literature results.

Besides the abovementioned limitations, numerous investigations have focused on the prospective impact of FDG-PET on staging and the choice of therapy in HL. ${ }^{19}$ These studies show that 11 to $41 \%$ of patients are upstaged by FDG-PET compared with conventional staging procedures and that 0 to $28 \%$ of patients are downstaged.

The proportion of patients for whom the FDG-PET findings would potentially have changed the treatment strategy ranges from 3 to $25 \%$. Similar to these aforementioned results, this study of a Brazilian population with a high rate of advanced stages of disease that have already been established by conventional methods indicates 
that $20.7 \%$ of these patients would have been upstaged and $10.9 \%$ would have been downstaged by implementation of the FDG-PET. If the stage were defined using the FDGPET results, it would have led to a change in the treatment strategy in $15.8 \%(13 / 82)$ of the patients, whereby seven patients would have received more aggressive treatment strategies and six patients would have received less aggressive treatment strategies.

\section{CONCLUSION}

The results of the present study indicate that FDG-PET has a high accuracy for the detection of nodal and extranodal $\mathrm{HL}$ and is superior to CT analysis alone. In addition, an excellent sensitivity for the detection of BM disease was observed using FDG-PET. The detection of additional lesions using FDG-PET imaging improves the accuracy of HL staging. Even in a Brazilian population that is characterized by a high rate of advanced stages of the disease using conventional methods, the FDG-PET most often upstages the disease and leads to a change in the treatment strategy in a significant number $(16 \%)$ of patients. However, changes in the clinical stage based only upon FDG-PET results should be performed with caution, and subsequent biopsy of the site in question may be necessary depending on the clinical context. The results of the modification of HL therapy based on initial FDG-PET findings are still an open question that will only be answered with controlled clinical trials.

\section{REFERENCES}

1. Lister TA, Crowther D, Sutcliffe SB, Glatstein E, Canellos GP, Young $\mathrm{RC}$, et al. Report of a committee convened to discuss the evaluation and staging of patients with Hodgkin disease. J Clin Oncol. 1989;7:16306.

2. Stein RS, Golomb HM, Diggs CH, Mauch P, Hellman S, Wiernik PH, et al. Anatomic substages of stage IIIA Hodgkin's disease. A collaborative study. Ann Intern Med. 1980;92:159-65.

3. Moog F, Bangerter M, Diederichs CG, Guhlmann A, Kotzerke J, Merkle E, et al. Lymphoma: role of whole-body 2-deoxy-2-[F-18]fluoro-Dglucose (FDG) PET in nodal staging. Radiology. 1997;203:795-800.

4. Schoder H, Meta J, Yap C, Ariannejad M, Rao J, Phelps ME, et al. Effect of whole-body 18F-FDG PET imaging on clinical staging and management of patients with malignant lymphoma. J Nucl Med. 2001;42:1139-43.

5. Naumann R, Beuthien-Baumann B, Reiss A, Schulze J, Hänel A, Bredow J,et al. Substantial impact of FDG PET imaging on the therapy decision in patients with early-stage Hodgkin's lymphoma. Br J Cancer. 2004;90:620-25.

6. Bangerter M, Moog F, Buchmann I, Kotzerke J, Griesshammer M, Hafner M, et al. Whole-body 2-[18F]-fluoro-2-deoxy-d-glucose positron emission tomography (FDG-PET) for accurate staging of Hodgkin's disease. Ann Oncol. 1998;9:1117-22.

7. Juweid ME, Stroobants S, Hoekstra OS, Mottaghy FM, Dietlein M, Guermazi A, et al: Use of positron emission tomography for response assessment of lymphoma: Consensus recommendations of the Imaging Subcommittee of the International Harmonization Project in Lymphoma. J Clin Oncol. 2006;10:25:571-8.
8. Spector N, Costa MA, Pulcheri W, Salgado RC, Nucci M, Andrade CA et al. CMOPP/ABV yields good results in a public hospital population with Hodgkin disease in Brazil. Cancer. 1993;71:2823-7.

9. Hutchings M, Loft A, Hansen M, Pedersen LM, Berthelsen AK, Keiding S, et al. Positron emission tomography with or without computed tomography in the primary staging of Hodgkin's lymphoma. Haematologica. 2006;91:482-9.

10. Jerusalem G, Beguin Y, Fassotte MF, Najjar F, Paulus P, Rigo P, et al Wholebody positron emission tomography using $18 \mathrm{~F}$-fluorodeoxyglucose compared to standard procedures for staging patients with Hodgkin's disease. Haematologica. 2001;86:266-73.

11. Weihrauch MR, Re D, Bischoff S, Dietlein M, Scheidhauer K, Krug B, et al. Whole-body positron emission tomography using 18F-fluorodeoxyglucose for initial staging of patients with Hodgkin's disease. Ann Hematol. 2002;81:20-5.

12. Menzel C, Döbert N, Mitrou P, Mose S, Diehl M, Berner U, et al. Positron emission tomography for the staging of Hodgkin's lymphoma - increasing the body of evidence in favor of the method. Acta Oncol. 2002;41:430-6.

13. Kwee TC, Kwee RM, Rutger AJN. Imaging in staging of malignant lymphoma: a systematic review. Blood. 2008;111:504-16.

14. Pracchia LF, Chaves AA, Cerci JJ, Soares Junior J, Meneghetti JC, Buccheri V. Metabolic test with fluorine-18-fluorodeoxyglucose in staging and detection of residual tumor or recurrence in Hodgkin lymphoma. Clinics. 2007;62:121-6. 
15. Wang J, Weiss LM, Chang KL, Slovak ML, Gaal K, Forman SJ, et al Diagnostic utility of bilateral bone marrow examination: significance of morphologic and ancillary technique study in malignancy. Câncer. 2002;94:1522-31.

16. Carr R, Barrington SF, Madan B, Carr R, Barrington SF, Madan B, et al. Detection of lymphoma in bone marrow by whole-body positron emission tomography. Blood. 1998;91:3340-6.

17. Schaefer NG, Strobel K, Taverna C, Hany TF. Bone involvement in patients with lymphoma: the role of FDG-PET/CT. Eur J Nucl Med Mol Imaging. 2007;34:60-7.
18. Jerusalem G, Silvestre RM, Beguin Y. Does 18F-FDG PET replace bone marrow biopsy (BMB) in patients with Hodgkin's disease (HD) or non-Hodgkin's lymphoma (NHL)? Blood 2002;100:768a.

19. Pakos EE, Fotopoulos AD, Ioannidis JP. 18F-FDG PET for evaluation of bone marrow infiltration in staging of lymphoma: a meta-analysis. J Nucl Med. 2005;46:958-63.

20. Vinnicombe SJ, Reznek RH. Computerised tomography in the staging of Hodgkin's disease and non-Hodgkin's lymphoma. Eur J Nucl Med Mol Imaging. 2003;30: Suppl 1:S42-55.

21. Podoloff DA, Macapinlac HA. PET and PET/CT in Management of the Lymphomas. Radiol Clin N Am. 2007;45:689-96. 